\title{
Prognostic significance of artemin and GFR $\alpha 1$ expression in laryngeal squamous cell carcinoma
}

\author{
CHAOBING GAO ${ }^{1}$, XINGWANG CHENG ${ }^{2}$, XIAOHONG LI $^{1}$, BUSHENG TONG $^{1}$, KAILE WU $^{1}$ and YEHAI LIU $^{1}$ \\ ${ }^{1}$ Department of Otorhinolaryngology, Head and Neck Surgery, The First Affiliated Hospital of Anhui Medical University, \\ Hefei, Anhui 230022; ${ }^{2}$ Department of Emergency, The First Affiliated Hospital of Bengbu Medical University, \\ Bengbu, Anhui 233030, P.R. China
}

Received January 19, 2014; Accepted June 5, 2014

DOI: $10.3892 /$ etm.2014.1821

\begin{abstract}
Artemin (ARTN) has been implicated in the development and progression of several human malignancies. However, the clinical and prognostic significance of ARTN and its receptors has not yet been investigated in human laryngeal squamous cell carcinoma (LSCC). Therefore, in the present study, the protein expression of ARTN and its receptor, namely GFR $\alpha 1$, was determined in 76 LSCC and 26 laryngeal polyp tissue samples using immunohistochemistry. Furthermore, the clinicopathological and prognostic significance of ARTN and GFR $\alpha 1$ expression was analyzed in patients with LSCC. The results revealed that the expression of ARTN and GFR $\alpha 1$ was significantly increased in LSCC compared with polyp tissue samples. Furthermore, the expression of ARTN and GFR $\alpha 1$ was positively associated with pTNM stage in LSCC. Kaplan-Meier survival analyses revealed a strong association between the expression of ARTN or GFR $\alpha 1$ and the survival of patients with LSCC. Correlation analysis demonstrated that the expression of ARTN was significantly correlated with the expression GFR $\alpha 1$. In conclusion, the results demonstrated that ARTN and GFR $\alpha 1$ may be useful predictors of disease progression and outcome in patients with LSCC.
\end{abstract}

\section{Introduction}

Laryngeal squamous cell carcinoma (LSCC) is one of the most common malignancies in the head and neck region, which leads to 350,000 mortalities worldwide each year $(1,2)$. Despite considerable advances in diagnosis and therapy, the survival of patients with LSCC remains poor. At present, there are no effective biomarkers for LSCC to assist early diagnosis or monitor patient prognosis. Furthermore, traditional prog-

Correspondence to: Professor Yehai Liu, Department of Otorhinolaryngology, Head and Neck Surgery, The First Affiliated Hospital of Anhui Medical University, 218 Jixi Road, Hefei, Anhui 230022, P.R. China

E-mail: liuyehai616@qq.com

Key words: artemin, GFR $\alpha 1$, laryngeal squamous cell, survival nostic markers, including tumor histological grade, clinical stages and lymph node metastasis, may not fully evaluate the patient's survival (3). Therefore, improving the understanding of the molecular mechanisms and gene alterations involved in the development and progression of LSCC may be helpful for the establishment of novel biomarkers to effectively monitor patients with LSCC.

Artemin (ARTN) is a growth factor that belongs to the glial cell line-derived neurotrophic factor (GDNF) family of ligands (GFL), which consists of four members, including GDNF, neurturin and persephin $(4,5)$. GFL family members (including ARTN) have been found to signal via interaction with one or more of the GDNF receptor $\alpha$ family (GFR $\alpha$ ), which is comprised of four members, GFR $\alpha 1-4$ (5-7). In addition to the role of neurotrophic factor (5-7), ARTN has also been found to have an oncogenic role in promoting tumor growth, migration, invasiveness and metastasis in a number of types of human cancer (8-14). For example, it has been demonstrated that the expression of ARTN is significantly increased in breast cancer tissues compared with normal breast tissues, and that high expression of ARTN is positively correlated with high tumor stage and poor survival in breast cancer (9). In endometrial cancer, high expression levels of ARTN have been observed be significantly associated with high tumor grade and myometrial invasiveness in clinical tissue specimens, and forced expression of ARTN has been demonstrated to increase tumor cell growth and invasiveness in vivo and in vitro (11). In addition, the expression levels of ARTN and its receptors have been observed to be upregulated in breast cancer and significantly associated with disease progression (15). Furthermore, co-expression of ARTN with its receptors has been found to produce synergistic increases in the odds ratio for survival in patients with breast cancer (15). These results suggest that ARTN with its receptors may have an important role in human solid tumors. However, to the best of our knowledge, the clinical impact and prognostic significance of ARTN or its receptor expression in human LSCC has not yet been investigated.

In the present study, the protein expression of ARTN and one of its receptors, GFR $\alpha 1$, in LSCC was determined using immunohistochemistry and the correlation between the expression levels, clinicopathological features and patient survival outcome was analyzed. The aim was to investigate 
whether ARTN and its receptors may be potential biomarkers of disease progression and prognosis in patients with LSCC.

\section{Materials and methods}

Patients and specimens. The patient population consisted of 76 consecutive patients with LSCC and 26 consecutive patients with benign polyp, who underwent surgery at the First Affiliated Hospital of Anhui Medical University (Hefei, China) between 2007 and 2009. None of the patients had undergone any chemotherapy or radiation therapy prior to the surgery, or had a previous diagnosis of carcinoma or a distant metastasis at the time of diagnosis. The pathohistological diagnosis and tumor histological grade of the patients was based on the World Health Organization (16). The pathological tumor staging (pstage) was determined according to the TNM classification of malignant tumors by the International Union Against Cancer (UICC, 2002) (17). The median time of patient follow up was 60 months. This study was approved by the institutional review board of the First Affiliated Hospital of Anhui Medical University (Anhui, China) and written, informed consent was obtained from all patients.

Immunohistochemistry. Formalin-fixed, paraffin-embedded tissues were collected from each patient and cut into 4- $\mu$ m-thick sections. Immunohistochemical analysis of ARTN and GFR $\alpha 1$ protein expression was performed using polyclonal antibodies against ARTN (1:100 dilution; R\&D Systems, Minneapolis, MN, USA) and GFR $\alpha 1$ (1:100 dilution; Santa Cruz Biotechnologies, Santa Cruz, CA, USA) using the peroxidase-conjugated streptavidin complex method (Histostain-SP kit; Zymed, San Francisco, CA, USA), as previously described (18).

Review and scoring. The results of the immunoreactivity of stained sections were reviewed and scored for expression of ARTN and GFR $\alpha 1$ using a light microscope (Olympus American Inc., Melville, NY, USA) by two pathologists in a blinded manner. The sections were scored based on the staining intensity and the percentage of cells with staining relative to the background (19). The evaluation of the extent of staining was based on the percentage of positive-stained cells among all the cells in the each case and scored from 0 to 4: 0 , $0 \%, 1,1-25 \%, 2,26-50 \%, 3,51-75 \%$ and $4,76-100 \%$. Similarly, the intensity of staining was based on the color of the certain cells in each case and scored from 0 to 3: 0, negative, 1 , weak, 2 , medium and 3, strong. The sum score of the intensity and extent of staining was used as the final score. Samples with a final score $>2$ were considered positive.

Statistical analysis. All statistical analyses of results were performed using SPSS software system for Windows (version 13.0; SPSS, Inc., Chicago, IL, USA). The chi-squared $\left(\chi^{2}\right)$ test was used to analyze the difference in the expression levels of ARTN and GFR $\alpha 1$ among different samples. Pearson's correlation coefficient was calculated to evaluate the association between the expression of ARTN and GFR $\alpha 1$. Kaplan-Meier curves were produced to determine patient relapse-free survival (RFS) and overall survival (OS) rates. The statistical differences in survival among subgroups were compared using
Table I. Expression of ARTN and GFR $\alpha 1$ in LSCC and polyp tissue specimens.

\begin{tabular}{lccc}
\hline & & \multicolumn{2}{c}{ Expression, $\mathrm{n}(\%)$} \\
\cline { 3 - 4 } Group & $\mathrm{n}$ & ARTN & GFR $\alpha 1$ \\
\hline LSCC & 76 & $41(53.9)^{\mathrm{a}}$ & $39(51.3)^{\mathrm{b}}$ \\
Polyp & 26 & $7(26.9)$ & $7(26.9)$ \\
\hline
\end{tabular}

${ }^{\mathrm{a}} \mathrm{P}=0.015$ and ${ }^{\mathrm{b}} \mathrm{P}=0.031$ vs. the polyp group. LSCC, laryngeal squamous cell carcinoma; ARTN, artemin; GFR $\alpha 1$, GDNF receptor $\alpha 1$.

the log-rank test. $\mathrm{P}<0.05$ was considered to indicate a statistically significant difference.

\section{Results}

Expression of ARTN and GFR 1 protein is upregulated in LSCC tissue samples. Immunohistochemistry was used to determine the expression of immunoreactive protein for ARTN and GFR $\alpha 1$ in a cohort of specimens. Positive signals were observed in the cytoplasm of the squamous cell carcinoma cells or squamous epithelium of polyp tissues (Fig. 1). As shown in Table I, 53.9 and $51.3 \%$ of LSCC samples were positive for ARTN and GFR $\alpha 1$, respectively, whilst only $26.9 \%$ of normal squamous epithelium from patients with polyp were positive for ARTN and GFR $\alpha 1(\mathrm{P}=0.015$ and $\mathrm{P}=0.031$, respectively).

Correlation between the expression of ARTN and GFR $\alpha 1$ and clinicopathological features of LSCC. The association of tumor expression of ARTN and GFR $\alpha 1$ with the clinicopathological features of LSCC was then investigated. As observed in Table II, the expression of ARTN and GFR $\alpha 1$ was significantly associated with advanced pTNM stage $(\mathrm{P}=0.024$ and $\mathrm{P}=0.006$, respectively). However, no significant association was observed between the expression of ARTN and GFR $\alpha 1$ and any other clinicopathological characteristics, including tumor site, tumor differentiation and tumor lymph node metastasis (all $\mathrm{P}>0.05$ ).

Correlation between ARTN and GFR 1 l expression and patient survival. To determine the prognostic significance of ARTN and GFR $\alpha 1$ expression in patients with LSCC, Kaplan-Meier analyses were performed to correlate the expression of these proteins with the RFS and OS of patients. As observed in Fig. 2, patients with LSCC whose tumors were positive for expression of ARTN had a significantly lower five-year RFS or OS compared with patients whose tumors were negative for ARTN $(\mathrm{P}=0.030$ and $\mathrm{P}=0.010$, respectively). Similarly, expression of GFR $\alpha 1$ protein also predicted a significantly lower five-year OS compared with patients whose tumors were negative for GFR $\alpha 1(\mathrm{P}=0.025)$. In addition, patients whose tumors expressed GFR $\alpha 1$ protein exhibited a lower RFS compared with patients whose tumors were negative for GFR $\alpha 1$ protein; however, this trend was not significant $(\mathrm{P}=0.071)$.

Correlation between ARTN and GFRal expression. Correlation analysis was then conducted to determine the 
Table II. Association of ARTN and GFR $\alpha 1$ expression with clinicopathological parameters from patients with LSCC.

\begin{tabular}{|c|c|c|c|c|c|}
\hline \multirow[b]{2}{*}{ Parameter } & \multirow[b]{2}{*}{$\mathrm{n}$} & \multicolumn{2}{|c|}{ ARTN } & \multicolumn{2}{|c|}{ GFR $\alpha 1$} \\
\hline & & Expression, n (\%) & P-value & Expression, n (\%) & P-value \\
\hline \multicolumn{6}{|l|}{ Age (years) } \\
\hline$\leq 60$ & 40 & $19(47.5)$ & 0.235 & $17(42.5)$ & 0.105 \\
\hline$>60$ & 36 & $22(61.1)$ & & $22(61.1)$ & \\
\hline \multicolumn{6}{|l|}{ Gender } \\
\hline Male & 70 & $39(55.7)$ & 0.291 & $37(52.9)$ & 0.358 \\
\hline Female & 6 & $2(33.3)$ & & $2(33.3)$ & \\
\hline \multicolumn{6}{|l|}{ Tumor site } \\
\hline Supraglottic & 22 & $14(63.6)$ & 0.521 & $15(68.2)$ & 0.131 \\
\hline Glottic & 45 & $23(51.1)$ & & $19(42.2)$ & \\
\hline Subglottic & 9 & $4(44.4)$ & & $5(51.3)$ & \\
\hline \multicolumn{6}{|c|}{ Tumor differentiation } \\
\hline Well & 28 & $17(60.7)$ & 0.122 & $16(57.1)$ & 0.681 \\
\hline Moderate & 32 & $19(59.4)$ & & $16(50.0)$ & \\
\hline Poor & 16 & $5(31.3)$ & & 7 (43.8) & \\
\hline \multicolumn{6}{|l|}{ pTNM stage } \\
\hline I-II & 35 & $14(40.0)$ & $0.024^{\mathrm{a}}$ & $12(34.3)$ & $0.006^{\mathrm{a}}$ \\
\hline III-IV & 41 & $27(65.9)$ & & $27(65.9)$ & \\
\hline \multicolumn{6}{|c|}{ Lymph node metastasis } \\
\hline Yes & 26 & $13(50.0)$ & 0.619 & $11(42.3)$ & 0.257 \\
\hline No & 50 & $28(56.0)$ & & $28(56.0)$ & \\
\hline
\end{tabular}

${ }^{\text {a }}<<0.05$. LSCC, laryngeal squamous cell carcinoma; ARTN, artemin; GFR $\alpha 1$, GDNF receptor $\alpha 1$.

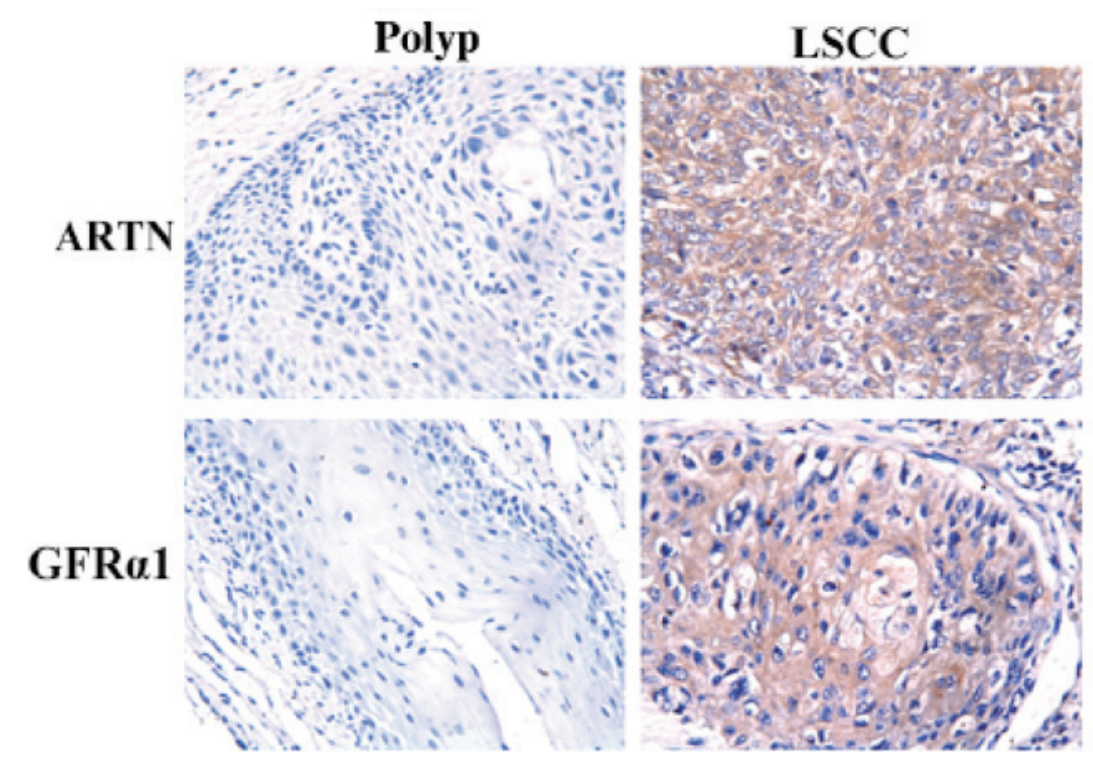

Figure 1. Expression of ARTN and GFR $\alpha 1$ in LSCC and polyp tissues specimens. Immunohistochemical analysis of ARTN and GFR $\alpha 1$ protein in LSCC and polyp. Low expression of ARTN and GFR $\alpha 1$ in polyp (left panels). High expression of ARTN and GFR $\alpha 1$ in LSCC (right panels). All images are counterstained with hematoxylin. Photomicrographs were captured at x200 magnification. LSCC, laryngeal squamous cell carcinoma; ARTN, artemin; GFR $\alpha 1$, GDNF receptor $\alpha 1$.

correlation between ARTN protein expression and the expression of GFR $\alpha 1$ protein in the same cohort of patients with LSCC. As was expected, Pearson's correlation analysis revealed that the expression of ARTN was significantly correlated with the expression of GFR $\alpha 1$ in these patients ( $\mathrm{rs}=0.527, \mathrm{P}=0.001)$. 

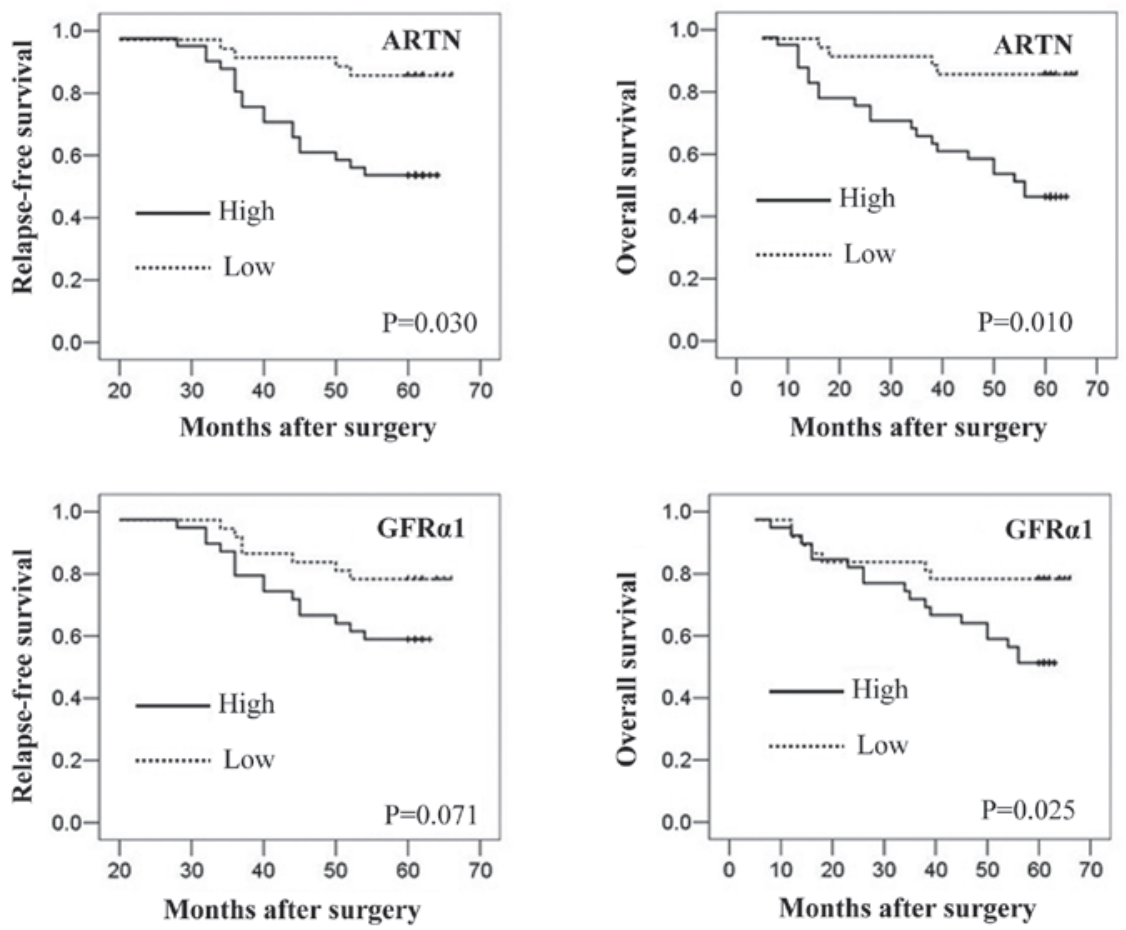

Figure 2. Correlation between ARTN and GFR $\alpha 1$ expression and patient survival. Kaplan-Meier analysis of the expression of ARTN and GFR $\alpha 1$ with the RFS and OS of patients with LSCC. LSCC, laryngeal squamous cell carcinoma; ARTN, artemin; GFR $\alpha 1$, GDNF receptor $\alpha 1$; RFS, relapse-free survival; OS, overall survival.

\section{Discussion}

In this study, it was observed that a neurotrophic factor, ARTN, was expressed at significantly higher levels in LSCC compared with the levels in benign laryngeal polyp tissue samples. Furthermore, the expression of ARTN was demonstrated to be significantly associated with high tumor stage and poor survival. In addition, previous studies have suggested that ARTN has a role in the development and progression of diverse human carcinoma $(9,10,12,20-22)$. In pancreatic ductal adenocarcinoma, ARTN has been reported to be highly expressed compared with normal pancreases, and stimulates the invasiveness of pancreatic cancer cells (13). Furthermore, the depletion of ARTN expression inhibits survival, invasion and anchorage-independent growth of both breast and endometrial cancer cells, while the forced expression of ARTN promotes these cellular behaviors $(9,11)$. Molecularly, ARTN stimulates survival and anchorage-independent growth of human non-small cell lung cancer cells by upregulating BCL-2 expression (12). In addition, ARTN stimulates estrogen receptor-negative breast cancer cell growth, migration and metastasis by upregulating TWIST1 expression and activating the AKT pathway (21). These studies are in accordance with the results from the present study, suggesting an oncogenic role of ARTN in the progression of human malignancies.

ARTN has been reported to bind to and activate GFR $\alpha 1$ (5), a member of the GDNF receptor $\alpha$ family. In order to determine whether GFR $\alpha 1$ mediates the effects of ARTN in LSCC, the protein expression of GFR $\alpha 1$ in LSCC and matched normal tissues was analyzed, and the correlation between ARTN and clinicopathological features and patient survival outcome was investigated. The results from the present study revealed that the expression of GFR $\alpha 1$ was increased in cancerous tissues compared with the expression level in normal tissues and was also significantly associated with high tumor stage and poor survival of patients, which indicates that GFR $\alpha 1$ has a similar role to that of ARTN in LSCC. Furthermore, Pearson's correlation analysis confirmed that the expression of GFR $\alpha 1$ has a significantly high correlation with ARTN expression. These results indicate that the functional effects of ARTN in the progression of LSCC may be mediated by GFR $\alpha 1$. Increased GFR $\alpha 1$ expression has been previously reported in breast cancer, and its expression is associated with tumor lymph node metastases and poor survival in patients $(8,15)$. In addition, the stimulation of GFR $\alpha 1$-positive breast cancer cells with GDNF has been previously demonstrated to enhance cell proliferation and survival in vivo (8). In human neuroblastoma, Yoong et al demonstrated that GFR $\alpha 1$ promotes neurite outgrowth in tumor cells via the activation of ERK1/2, Rac1 and Cdc42 (23).

In conclusion, to the best of our knowledge, this study demonstrates for the first time the altered expression of ARTN and GFR $\alpha 1$ in LSCC and the association of the expression levels of these proteins with high stage disease and poor survival outcome for patients. The expression levels of ARTN and GFR $\alpha 1$ protein may therefore be useful as prognostic markers in LSCC. Whether other receptors of ARTN may also mediate its effects on the progression of LSCC remains to be determined.

\section{Acknowledgements}

This study was supported in part by a grant from the First Affiliated Hospital of Anhui Medical University and by a key 
program of the Educational Department in Anhui, China. (grant no. KJ2012A162).

\section{References}

1. Jemal A, Bray F, Center MM, Ferlay J, Ward E and Forman D: Global cancer statistics. CA Cancer J Clin 61: 69-90, 2011.

2. Chu EA and Kim YJ: Laryngeal cancer: diagnosis and preoperative work-up. Otolaryngol Clin North Am 41: 673-695, 2008.

3. Tang XB, Shen XH, Li L, Zhang YF and Chen GQ: SOX2 overexpression correlates with poor prognosis in laryngeal squamous cell carcinoma. Auris Nasus Larynx 40: 481-486, 2013.

4. Airaksinen MS, Holm L and Hätinen T: Evolution of the GDNF family ligands and receptors. Brain Behav Evol 68: 181-190, 2006.

5. Baloh RH, Tansey MG, Lampe PA, et al: Artemin, a novel member of the GDNF ligand family, supports peripheral and central neurons and signals through the GFRalpha3-RET receptor complex. Neuron 21: 1291-1302, 1998.

6. Airaksinen MS and Saarma M: The GDNF family: signalling, biological functions and therapeutic value. Nat Rev Neurosci 3 . 383-394, 2002

7. Airaksinen MS, Titievsky A and Saarma M: GDNF family neurotrophic factor signaling: four masters, one servant? Mol Cell Neurosci 13: 313-325, 1999.

8. Esseghir S, Todd SK, Hunt T, et al: A role for glial cell derived neurotrophic factor induced expression by inflammatory cytokines and RET/GFR alpha 1 receptor up-regulation in breast cancer. Cancer Res 67: 11732-11741, 2007.

9. Kang J, Perry JK, Pandey V, et al: Artemin is oncogenic for human mammary carcinoma cells. Oncogene 28: 2034-2045, 2009.

10. Pandey V, Jung Y, Kang J, et al: Artemin reduces sensitivity to doxorubicin and paclitaxel in endometrial carcinoma cells through specific regulation of CD24. Transl Oncol 3: 218-229, 2010.

11. Pandey V, Qian PX, Kang J, et al: Artemin stimulates oncogenicity and invasiveness of human endometrial carcinoma cells. Endocrinology 151: 909-920, 2010.

12. Tang JZ, Kong XJ, Kang J, et al: Artemin-stimulated progression of human non-small cell lung carcinoma is mediated by BCL2 Mol Cancer Ther 9: 1697-1708, 2010.
13. Ceyhan GO, Giese NA, Erkan M, et al: The neurotrophic factor artemin promotes pancreatic cancer invasion. Ann Surg 244: 274-281, 2006

14. Li S, Li Z, Guo F, et al: miR-223 regulates migration and invasion by targeting Artemin in human esophageal carcinoma. J Biomed Sci 18: 24, 2011

15. Wu ZS, Pandey V, Wu WY, Ye S, Zhu T and Lobie PE: Prognostic significance of the expression of GFR $\alpha 1$, GFR $\alpha 3$ and syndecan-3, proteins binding ARTEMIN, in mammary carcinoma. BMC Cancer 13: 34, 2013.

16. Thompson L. World Health Organization classification of tumours: pathology and genetics of head and neck tumours. Ear Nose Throat J 85: 74, 2006

17. Sobin L and Wittekind C (eds). TNM classification of malignant tumours. 6th edition. Wiley-Liss, New York, NY, p39, 2002

18. Yang Q, Liu Y, Huang Y, et al: Expression of COX-2, CD44v6 and CD147 and relationship with invasion and lymph node metastasis in hypopharyngeal squamous cell carcinoma. PLoS One 8: e71048, 2013

19. Masunaga R, Kohno H, Dhar DK, et al: Cyclooxygenase-2 expression correlates with tumor neovascularization and prognosis in human colorectal carcinoma patients. Clin Cancer Res 6: 4064-4068, 2000.

20. Banerjee A, Qian P, Wu ZS, et al: Artemin stimulates radio- and chemo-resistance by promoting TWIST1-BCL-2-dependent cancer stem cell-like behavior in mammary carcinoma cells. J Biol Chem 287: 42502-42515, 2012.

21. Banerjee A, Wu ZS, Qian P, et al: ARTEMIN synergizes with TWIST1 to promote metastasis and poor survival outcome in patients with ER negative mammary carcinoma. Breast Cancer Res 13: R112, 2011.

22. Banerjee A, Wu ZS, Qian PX, et al: ARTEMIN promotes de novo angiogenesis in ER negative mammary carcinoma through activation of TWIST1-VEGF-A signalling. PLoS One 7: e50098, 2012.

23. Yoong LF, Wan G and Too HP: GDNF-induced cell signaling and neurite outgrowths are differentially mediated by GFRalpha1 isoforms. Mol Cell Neurosci 41: 464-473, 2009. 